\title{
Redefining medical education by boosting curriculum with artificial intelligence knowledge
}

\section{Is Al a threat to doctors?}

The answer is neither simple nor direct. We cannot honestly profess to know the answer to this complex query, but throughout this textbook, you will find our sincere observations regarding this multi-faceted topic. It is clear that in various healthcare areas, such as medical areas and fraud, AI will be transformational. It will herald new, innovative, and faster ways to discover drugs and help manage cancer patients. Virtual assistants, sensor devices, and personalized medicine are clearly here to stay, and they will modify disease management. Imaging and cardiac rhythm management has already witnessed seismic changes as has our approach to mental health.

As opposed to these positive developments, we remain concerned about ethics, the "black box" phenomenon, privacy issues, and the overwhelming apprehension of "machines replacing us."

We remain hopeful that our textbook and this chapter will restore balance in your individual analysis of AI's role in medicine.

For physicians, a concern about the continuity of their profession and its impact on medical education is paramount. We devote this chapter to review the role that $\mathrm{AI}$ is likely to play in medical education.

\section{Background of the medical school curriculum}

In 1910, Abraham Flexner published The Flexner Report. After visiting 155 medical schools across the United States and Canada, he established the biomedical model as the gold standard of medical training. ${ }^{1}$ Among other things, he created a standardized four-year curriculum, recommended a minimum qualification for admittance, and establishes an accreditation process. ${ }^{2}$ Previous to his report, the majority of medical schools had been founded merely for profit reasons and thus went about their business without any set of rules for admission or accreditation. Flexner's influence still guides the current curricular reform, and more than a century later, we still believe the fundamental aims proposed by him are relevant. However, we must also consider that to restructure today's education track optimally, it is necessary to embrace new technologies.

\section{Making changes to the medical school curriculum}

In the recent past, medical curriculum reformations were made to meet requirements urged by the accreditation system. These changes are essential, but they are not enough. As Wartman and Combs state in their 2019 article, a genuinely impactful reimagining cannot correctly take place within the existing regulatory structure. ${ }^{3}$ As such, adjusting the accreditation and licensing framework should be strongly considered. ${ }^{4}$ The 21 st-century curriculum should include components that strengthen the physician's capacity to practice with more precision in such a data-rich, rapidly advancing environment that is supported by ever-improving AI. Since the vast amount of available medical information has literally surpassed our brains' storage capacity, teaching medical students to acclimate to and embrace AI applications should be a central focus of today's curricular reform.

Traditionally, medical education has revolved around memorization. As a result, students and physicians are spending much
Volume 13 Issue 5 - 2020

\author{
Sameer Mehta, Daniel Vieira, Samantha \\ Quintero, Daniela Bou Daher, Floralba \\ Duka, Hudson Franca, Jhonny Bonilla, \\ Andreea Molnar, Catalina Molnar, David \\ Zerpa, María Fernanda Fleming Díaz, Andrea \\ De La Hoz, María José González, Michelle \\ Carraquel, Rosangel Salazar, Daniela Fawcett, \\ Gianfranco Bittar, Sonya Stoica, Eliana Mujica, \\ Mario Zevallos, Thais Lee Proenza, Maria \\ Fernanda Valles, Maria Hernandez, Maritza \\ Muir, Daniela Ramirez, Carlos Mondragon, \\ Carmen Sanchez, Daniel Pinos, Lorena \\ Pisana, Maria Angelica Marin, Claudia Lopez \\ Lumen Foundation, USA
}

Correspondence: Sameer Mehta, Lumen Foundation, 9386 SW IST ST, USA, Tel 7543026633, Email davie889@hotmail.com

Received: September 30, 2020 | Published: October 13, 2020

of their time attending to tasks that could be more easily completed by a machine. For instance, first-year residents devote an average of 16 out of every 24 hours worked to indirect patient care, of which at least 10 hours are dedicated to interacting with medical records. They spend a mere three hours (or less) engaged in direct patient care. ${ }^{5}$ Considering that healthcare should focus on the patient and not their records, this is entirely unacceptable. Another study concluded that primary care physicians (PCPs) spend approximately six hours a day just interacting with the electronic health records (EHR) during and after clinic hours. ${ }^{6}$ This monstrous proportion contributes to work-life imbalance, dissatisfaction, high attrition rates, and a burnout rate that exceeds $50 \%{ }^{7}$ PCPs feel burdened by mostly menial administrative tasks that are antithetical to the reasons they chose their profession in the first place while adding little value to patient care. ${ }^{8}$ In a study published by Neumann et al., distress associated with life and job dissatisfaction is a crucial reason for medical students and residents displaying a decline in empathy: anxiety, tension, and stress can significantly reduce one's ability to relate and sympathize. ${ }^{9}$

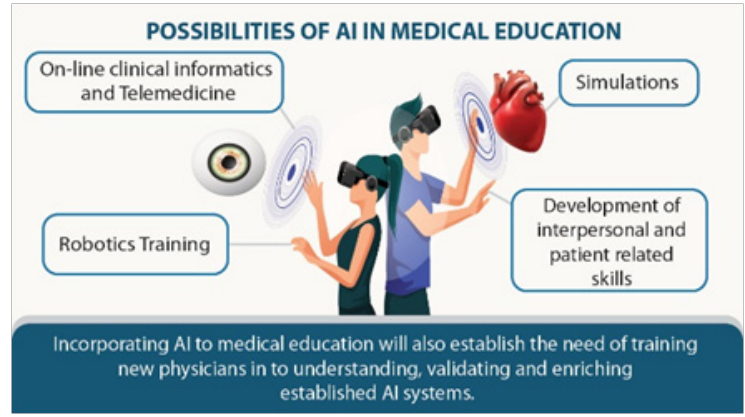

Crucial concepts like communication, shared decision making, leadership, team building, and empathy make up typically only a minor part of the medical school curriculum. ${ }^{10}$ Instead of focusing on 
building attributes that are critical features in physicians, students are still being taught how to memorize more efficiently.

It is time to allow ourselves to accept help. It is time to stop taking work home. Artificial intelligence is the relief physicians have long needed. As the amount of material published about artificial intelligence has steeply increased since 2010, it is evident that the biomedical research community has already embraced it as a necessary tool. Nonetheless, it is a glaring fact that the volume of AI-related publications geared towards undergraduate and graduate medical students have remained relatively unchanged over that same period. ${ }^{11}$ Sooner rather than later, medical education administrators will have to bite the bullet and search for ways to integrate this ascending technology into their curriculums. We believe that time is now.

In his book published in 2009, David E. Kern presented six steps often used as a guide for curricular design. These are: 1. Problem identification and general needs assessment; 2. Needs assessment for targeted learners; 3. Goals and objectives; 4. Educational strategies; 5. Implementation; and 6. Evaluation and feedback. ${ }^{12}$ In his book published in 2009, David E. Kern presented six steps often used as a guide for curricular design. These are: 1. Problem identification and general needs assessment; 2 . Needs assessment for targeted learners; 3. Goals and objectives; 4. Educational strategies; 5. Implementation; and 6. Evaluation and feedback. ${ }^{12}$ In terms of AI, the problem has already been identified so far, targeted learners' needs have already been assessed, and goals and objectives have already been defined. As we move forward, education strategies must be precisely designed, and their implementation, though imminent, will be far from an easy task.

There is a growing gap in training between emerging technologies and our current medical education model. It does not prepare young physicians to work collaboratively with AI. There are several reasons for this, not the least of which being that the curriculum carries a strong emphasis on preparing undergraduate students for the National Board Medical Examinations. In addition, accreditation is a major concern, faculties often resist change, and most professors lack the training and expertise needed to lead courses around such quickly advancing and groundbreaking technology. The speed of innovation means that the skillsets of countless teachers are rapidly becoming outdated or obsolete. So, preparing as soon as possible for a shift into a new education paradigm is imperative.

As Vijaya Kolachalama suggested in his article, ${ }^{11}$ we should be moving in a direction where educators are data scientists who use every day clinical examples to help make students more comfortable with AI, instead of dwelling on tedious definitions or equations. In March 2019, for the first time, Duke University offered a training course called "Machine Learning School for the School of Medicine" (MLS-SOM). It served as an introduction for its faculty and staff to machine and deep learning techniques. This sort of approach is crucial to keeping medical schools on the right track and vital to preparing faculty, and consequently, students for the near future. Harvard has recently taken another pioneering step with its Privacy Tools Project, which provides confidential data useful to researchers preserving the patients' valuable privacy. ${ }^{12}$

It is so important to evolve the way we teach our future physicians because the impact of trailblazing technology is already being felt across multiple medical specialties. This is despite doctors that largely maintain the belief that medicine is resistant to major disruptions by technology. ${ }^{13}$ According to Eric Topol's 2019 report, genomics, digital medicines, AI, and robotics will drastically alter the roles and functions of clinical staff over the next two decades, but, in various areas, the application of AI in medicine is already happening. ${ }^{14}$ Personal health virtual assistants can be combined with apps to deliver medication alerts, which will increase patient adherence to their treatment plans and thus avoid unnecessary hospital admissions. It is also possible to collect variables that can provide personalized risk scores, and the resulting higher efficiency that AI brings will allow specialists like radiologists to perform more value-added tasks more often. ${ }^{15}$

\section{The machines are not the problem. Rather, they are the solution}

Instead of perceiving advancing technologies with fear or as our enemies, we should learn how to leverage them as our allies It is so important that physicians reinvent themselves and adapt to 21stcentury medicine complexities, which is markedly better and more efficient than it has ever been. Applications of machine learning to highly specific cognitive tasks will increase not just the performance but also the value of health professionals. It will not replace them. ${ }^{16}$

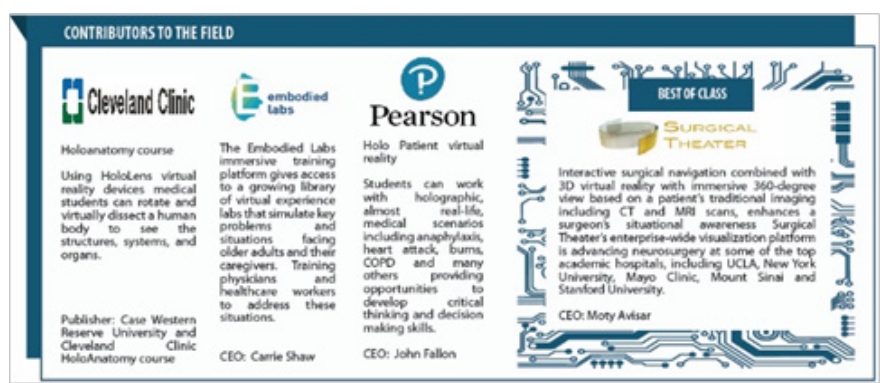

Human abilities like the tone of voice, empathy, body language interpretation, leadership, and shared decision-making are irreplaceable, critical components of medicine, and they always will be. ${ }^{10}$ Machines, when used properly, will serve to free up physicians so that they may incorporate more compassion into their practice, skill, and value that is essential but has been all but lost to the minutiae. The doctor-patient relationship is so much more than physical examinations and prescriptions. It is a human bond. It will remain that way, no matter how advanced technology gets. AI will provide the physician with space and time to reconstruct that connection -- finally, they will be able to look in their patients' eyes again when they are talking instead of sitting behind a computer screen typing every detail discussed. AI will help maximize the precious time during each visit. Patients will feel more taken care of. They will feel that their needs are being addressed with the attention they deserve. Restoring that time can only happen if we create a system that allows for such a level of relatability. Artificial intelligence is the way, and to build a balanced system, physicians will need proper training early on. ${ }^{17}$

Research has determined that doctors' job satisfaction improves with more meaningful patient communication and the delivery of better care ${ }^{10,18}$ Accordingly, it is paramount that we recognize and work to adjust the constraints in the physician-patient relationships that we have today. During the most recent AI World Conference in China, Elon Musk and Jack Ma, two icons in the areas of technology and artificial intelligence, shared their thoughts about the current and future effects of our advancements. Ma firmly believes that in the next 10-20years, efforts should shift towards education reform. The billionaire is advocating for less (but far more productive) hours spent at work with the help of AI. ${ }^{19}$ Musk evaluates that as the current education system is slow and humans are "dumb," as a unified race, we can be smarter than we are right now if we utilize the resources we have discovered to our advantage. ${ }^{19}$ 


\section{Integrating Al into education - undergraduate studies and pre-medicine track}

Literature about education in the future emphasizes that machines will replace many teachers. Medical schools will need to integrate education about AI into their curriculum to familiarize and form their students. Let us unpack this and examine how exactly we might integrate $\mathrm{AI}$ into an improved curriculum.

\section{Beginning with pre-med}

Since the long path to becoming a physician starts as early as undergraduate education, the pre-med track should be the first to implement changes into its landscape by installing requirements for the completion of data science, machine learning, and leadership courses. Calculus and statistics courses are already mandatory. Even though their use in practice is minimal, intelligent algorithms in AI build on those subjects, so if we do not provide students with the foundation from data science, they will not be able to synthesize certain information into something meaningful and applicable. Data science and machine learning courses tie calculus and statistics into a closed-loop, solidifying the real purpose and value in completing those requisites.

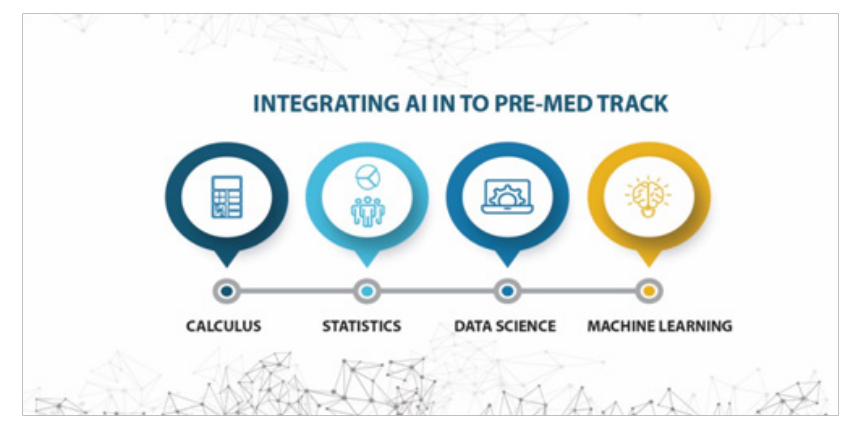

Leadership courses will also be necessary. Because the advancements in healthcare are so novel and groundbreaking, we will need to balance this evolution with passionate and competent students and doctors who can organize influential groups and assist in trialing all the different technologies. ${ }^{20}$ Students will begin to understand how feeding more and more data into a system works towards making it more precise and accurate at the pre-med level. We will emphasize excellent quality data mining and machine learning basics for these future doctors. Teaching them how to recognize all biases when feeding data into a system, showing them how to identify machine learning mechanisms used for various scenarios, and imparting general coding basics in well-used programs will help build strong foundations.

Since we want students to learn data science early in their premed track, we need teachers who are well-versed in this knowledge. Thus, we should enable data scientists to train current undergraduate professors who are associated with pre-medical courses. Once the system is in place, that training will progressively decrease as our future teachers will already be familiar with $\mathrm{AI}$ and machine learning. Of course, the evolution of the intelligent sciences is increasing and -- just as with traditional medicine -- professionals will have to keep updated.

\section{Integrating Al into education - medical schools and medical students}

Continuing to educate about AI throughout the medical school will transform the basic comprehension foundation into a dynamic understanding of its intricacies. Ideally, the medical school curriculum should encompass clinical informatics (the study of technology and its effects on healthcare) and telemedicine. Before outlining solutions for incorporating AI education, we should first go over our goals for medical students:

The overarching, primary goal is to produce a proactive medical student who is self-aware and cognizant of patients, e-patients, and the evolving healthcare environment. Our students must be sound in the narrative, mechanistic, and mathematical thinking. He or she should possess tools that help them leverage changes within medicine, organize and lead groups, and assist directly in designing and trialing EMR and AI systems, all while ensuring that they meet appropriate ethical, medical, and practical standards. ${ }^{21,22}$

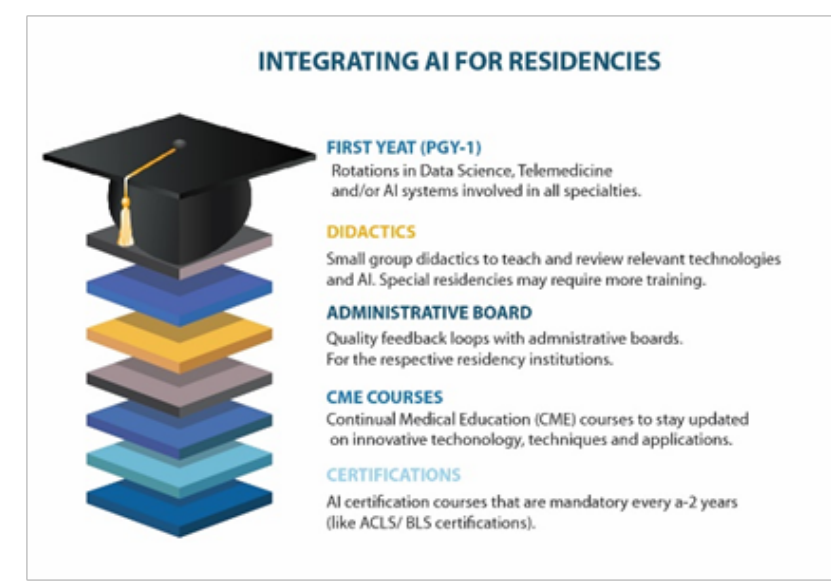

E-patients are patients that scour the internet for medical information and make decisions based on what they discover. They are deeply engaged in their healthcare and use a variety of electronic sources. Teaching medical students about the e-patient means diving into internet growth and usage patterns, examining the role the internet plays in patients' lives, pointing patients towards reliable databases, illuminating research methods, and many other facets. ${ }^{23}$

Narrative thinking is crucial because humans have a level of transcendental understanding that machines do not, which we use to discern others' intentions by using stories. Narrative thinking is our basis for understanding other people's minds via comparisons and overlaps between one another's experience. Conversely, the science of medicine employs a combination of mechanistic and mathematical thinking. Abstract concepts needed for hypothesizing and interpreting observations generated from experiments to fall under the umbrella of mechanistic thinking. Mathematical thinking is what will shape our future as it employs data science webbed with algorithms, statistics, and probabilities. ${ }^{22}$

What we want for medical students is what we want for medical doctors. Remembering that the foundation for skills that physicians have today grows out of previous experiences introduced early on along their journeys. Students should participate in frequent and diverse experiential learning opportunities, discussions, hands-on practice, self-awareness and reflection exercises, and mentorship and role-modeling. ${ }^{24}$ With that in mind, we propose the following integrations into the medical school curriculum (average four-year program):

The first two years (basic sciences) should consist of aspects of medical humanities, interprofessional team-based learning, healthcare leadership, telemedicine, and clinical informatics: 
a. The medical humanities portion will encompass humanities, social sciences, literature, sociology, psychology, high-level discourse, and history. This will provide a foundation for the medical student's narrative mindset while training them in deep counseling for patients and on the statistics of different treatment modalities and management. ${ }^{20}$

b. Interprofessional team-based learning for research and projects, as proposed by Chen et al., involves two or more professions collaborating in a deliberate effort to prepare students for the future reality of multidisciplinary team-based care. ${ }^{24}$

c. Healthcare leadership teaches students to facilitate, organize, and lead groups toward desired outcomes. ${ }^{25}$

d. Clinical informatics focuses on data science, machine learning, big data analysis, biostatistics, epidemiology, and evidencebased medicine..$^{25,26}$ Telemedicine will integrate under clinical informatics, and it will guide students in the care of e-patients and patients who benefit from telemedicine.

We propose to create an online clinical informatics and telemedicine course. Students must complete this course before starting their rotations, over the summer months, or during times when they have breaks from school. Brown et al. talk about the success of the online clinical informatics course they implemented and how it is self-paced learning. ${ }^{27}$ Self-paced learning will allow students to enjoy their free time and avoid burnout while simultaneously augmenting their education via this online course.

The second two years (clinical rotations) should incorporate didactics courses and a core rotation that boasts a substantial telemedicine/clinical informatics element. To promote small group learning, the didactics courses should be held in small group sessions. ${ }^{28}$ The telemedicine/clinical informatics rotation should teach students about various telemedicine applications and systems, touch on the use of big data analysis and its practical application (such as in EHR), and go into detail about the use of certain AI technologies that have been built specifically for fields like radiology, surgical robotics, etc. An example described by Khumrin et al. ${ }^{29}$ used trained e-learning systems that had students interact with virtual patients. This developed diagnostic reasoning skills for use in cases in which patients complained of acute abdominal pain. ${ }^{28}$ Not only will medical students see merit in using such tools, but they will also become more adept than ever before. If we want faster innovation and more equipped professionals, our students' exposure must happen as early as possible.

All this proposed, computers are not a "be all end all" solution. They will never be able to replace a self-reflective medical expert who is aware of his or her strengths and limitations, as well as the strengths and weaknesses of his or her patients. There is simply no substitute for physician-scientists who can integrate and master the art of medicine, scientific-mechanistic thinking, and scientific-mathematical thinking. However, to maximize potential and efficiency, the landscape of big data requires innovative approaches to the handling and organization of an often overwhelming glut of information. ${ }^{22}$

\section{Integrating Al into education - medical residents}

Residents must be more receptive to the influences of AI that are already making a broad impact in medicine. Providing them compulsory experiences with telemedicine and $\mathrm{AI}$ interactions during their first four years will broaden their base and encourage their acceptance of these innovations. Postgraduate Year 1 (PGY-1) intern year residents should complete initial rotations for telemedicine/data/ AI regardless of specialty. These individuals should receive significant exposure to the technology and AI around them, just as we proposed for medical students amid their clinical rotations At their advancing level, residents have more influence on the integration and acceptance of new technologies and procedures. They can sit on boards at their respective institutions and likely facilitate change since they are out on the front lines. The time spent in the hospital being handson and patient-facing provides residents with constant immersion in their field. As such, their administrations look to them for valuable feedback about the quality of newly introduced innovations.

Residents also attend regular didactic sessions that are scheduled by chief residents, directors, and attendings. Just as we advised for the better education of medical students, we encourage programs to break into small groups for these didactics. We also urge interprofessional team-based learning that incorporates residents from different specialties to collaborate on overall improvements, projects, and goals.

a. PGY-1 rotations in data science/telemedicine/AI systems for all specialties.

b. Interprofessional team-based learning for systems and projects.

c. Small group didactics to teach and review technologies and AI.

d. Quality feedback loops with administrative boards for each respective institution.

e. CME courses to stay updated on innovative technology, techniques, and applications.

f. AI certification courses that are mandatory requirements (similar to ACLS/BLS certifications).

Specialty programs must provide their residents with appropriate training for any robotics that they intend to use during procedures. Specialty residencies like robotic surgery may require several layers of didactics training. In a study by Winter et al., surgical residents from Penn State completed online modules, a literature review, ten hours of simulation training, and then a 90-minute bedside session involving all components of the DaVinci Platform in a non-operative setting..$^{29}$ After fulfilling these intentionally sequential requirements, residents had the experience necessary to operate the DaVinci platform technology console and could first assist in a surgical case. The program divided the instruction into trainee-directed and expertdirected training.

Even for non-surgical residents, the opportunity to conduct simulations, carry a light caseload and study the software of the DaVinci Platform will be rewarding. More familiarity with new technologies built off existing platforms will decrease any future learning gaps for all.

\section{Integrating Al into education - physicians, hospitals, and practice}

Whether a physician is privately practicing, part of a group practice, or working for a hospital, he or she has an obligation to stay current with innovative AI and telemedicine technologies. Creating a feedback loop with the administration within their institutions will be more challenging at this level. If a physician takes on more 
responsibility and holds a role on the board, this will be more easily achieved. If not, there will still be many ways to keep physicians up to speed with the evolving landscape of healthcare. Here are some we propose:

a. Quality feedback loops with administrative boards for the hospital/institution, if applicable.

b. Completion of CME courses that will help them stay updated on innovative technology, techniques, and applications.

c. AI certification courses that are annual mandatory requirements (like ACLS/BLS certifications).

d. Organized AI/telemedicine workshops at conferences, hospitals, and group practices.

It is crucial for doctors, and any individual on track to become one, to stress keeping current with machine learning and AI. The Accreditation Council for Graduate Medical Education (ACGME) identified six domains that comprise physician competence. They include medical knowledge, patient care, communication, practicebased learning, systems-based practice, and interpersonal relationships. Recent studies utilized machine learning and AI to assess how adept surgery and radiology physicians are according to those guidelines. In those specific specialties, AI and machine learning have a more massive impact. As time goes on, it will be important to educate physicians about the potential these tools have to help them become even more competent, while supplementing care, supporting training, and increasing patient safety. More current knowledge will build upon itself and facilitate widespread adoption and further research. ${ }^{30}$

\section{Conclusion}

As changes are implemented into the various phases, from the premedical student to resident and later to a full-fledged physician, there will be supplementary subjects that will need broadening -- ethics, epidemiology, biostatistics, policy, and medical humanities to name a few. Along with new developing technologies, new dilemmas will arise. When they do, they will likely be in one of the subjects listed above, and they must be addressed accordingly.

Initially, we will undoubtedly run into obstacles when instituting the suggested changes into curriculums. These speedbumps may be issues with early adoption, resistance to technology, technological limitations, snail-paced change in the accreditation process, and difficulties educating staff/professors/attendings on the technology. Those issues aside, as the demand for data science increases, the supply of actual data scientists who are willing and able to train others may prove to be the most limiting factor in adoption and innovation within the field. Though necessary, it will certainly not be smooth or unencumbered.

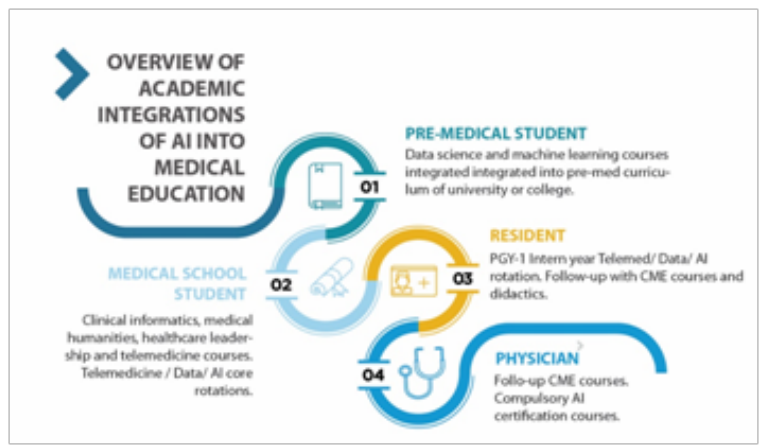

We mentioned that establishing a strong foundation for doctors to build upon requires the integration of elements early in their education. Thus, we have proposed a draft of several ways to integrate AI education at different levels to become a physician. Eventually, their technical acumen will push the ceiling for machine learning and artificial intelligence. This inevitable evolution of healthcare and medicine will bring along with it a new pedigree of physicians with newly restored compassion and empathy for all patients. After all, and very ironically, machines will be what make medicine human again.

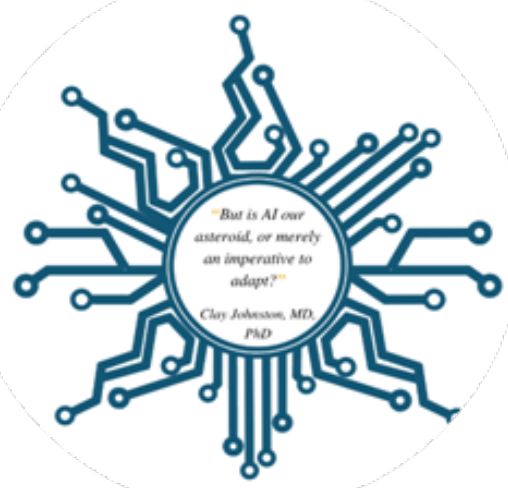

Acknowledgments

None.

\section{Conflicts of interest}

Author declares that there are no conflicts of interest.

\section{Funding}

None.

\section{References}

1. Duffy T P. The Flexner Report-100 Years Later. Yale J Biol Med. 2011;84(3):269-276.

2. Irby D M, Cooke M, Brien BCO. Calls for reform of medical education by the Carnegie Foundation for the Advancement of Teaching: 1910 and 2010. Acad Med. 2010;85(2):220-227.

3. Gordon JA, Hayden EM, Ahmed RA, et al. Early bedside care during preclinical medical education: Can Technology-Enhanced Patient Simulation Advance the Flexnerian Ideal ? Acad Med. 2010;85(2):370377.

4. Wartman SA, Combs CD. Reimagining Medical Education in the Age of AI. AMA J Ethics. 2019;21(2):E146-E152.

5. Wartman SA, Donald Combs C. Medical education must move from the information age to the age of artificial intelligence. Acad Med. 2019;93(8):1107-1109.

6. Arndt BG, Beasley JW, Watkinson MD, et al.Tethered to the EHR: Primary care physician workload assessment using EHR event log data and time-motion observations. Ann Fam Med. 2017;15(5):419-426.

7. Chaiyachati KH, Shea JA, Asch DA, et al. Assessment of Inpatient Time Allocation among First-Year Internal Medicine Residents Using TimeMotion Observations. JAMA Intern Med. 2019;179:60-67.

8. Densen P. Challenges and opportunities facing medical education. Trans Am Clin Climatol Assoc. 2011;122:48-58.

9. Neumann M, Edelhäuser F, Tauschel D, et al. Empathy decline and its reasons: A systematic review of studies with medical students and residents. Acad Med. 2011;86(8):996-1009. 
10. Johnston SC. Anticipating and Training the Physician of the Future: The Importance of Caring in an Age of Artificial Intelligence. Acad Med. 2018;93(8):1105-1106.

11. Kolachalama VB, Garg PS. Machine learning and medical education. NPJ Digit Med. 2018 S;1:54.

12. Shaw J. The Privacy Tools Project. USA: Harvard Magazine; 2017.

13. Amarin J, Borgan S. The Pervasive Culture of Abuse in Medical Education: A Focus on Developing Countries. Academic Medicine. 2017;92(5):578-579.

14. Topol E 2019 The Topol Review - NHS Health Education England.

15. Pesapane F, Codari M, Sardanelli F. Artificial intelligence in medical imaging: threat or opportunity? Radiologists again at the forefront of innovation in medicine. Eur Radiol Exp. 2018;2(1):35.

16. Li D, Kulasegaram K, Hodges BD. Why We Needn’t Fear the Machines: Opportunities for Medicine in a Machine Learning World Acad Med. 2019;94(5):623-625.

17. Topol EJ. High-performance medicine: the convergence of human and artificial intelligence. Nat Med. 2019;25(1):44-56.

18. Ommaya AK, Cipriano PF, Hoyt DB, et al. Care-Centered Clinical Documentation in the Digital Environment: Solutions to Alleviate Burnout. NAM Perspectives. 2018;10.31478/201801c.

19. Hickey A. Big Ideas: The Wild Thoughts of Elon Musk and Jack Ma. The morning Brew. 2019.

20. Sanal MG, Paul K, Kumar S, et al. Artificial intelligence and deep learning: The future of medicine and medical practice. $J$ Assoc Physicians India. 2019;67(4):71-73.

21. Masters K. Artificial intelligence in medical education. Med Teach. 2019;41(9):976-980.
22. Marchalik D. The Return to Literature - Making Doctors Matter in the New Era of Medicine. Acad Med. 2017;92(12):1665-1667.

23. De Leon J. Teaching Medical Students How To Think: Narrative, Mechanistic and Mathematical Thinking. Actas Esp Psiquiatr. 2018;46(4):133-145.

24. Masters K. Preparing medical students for the e-patient. Med Teach. 2017;39(7):681-685.

25. Chen J. Playing to our human strengths to prepare medical students for the future. Korean J Med Educ. 2017;29(3):193-197.

26. Hamdy H. Medical College of the Future: from Informative to Transformative. Med Teach. 2018;40(10):986-989.

27. Park SH, Do KH, Kim S, et al. What should medical students know about artificial intelligence in medicine? J Educ Eval Health Prof. 2019;16:18.

28. Brown HM, Latimer KS, Erikson LE, et al. Detection of persistent Cytauxzoon felis infection by polymerase chain reaction in three asymptomatic domestic cats. J Vet Diagn Invest. 2008;20(4):485-458.

29. Khumrin P, Ryan A, Judd T and Verspoor K. Diagnostic machine learning models for acute abdominal pain: Towards an e-learning tool for medical students Students. Health Technol Inform. 2017;245:447451 .

30. Winder JS, Juza RM, Sasaki J, et al. Implementing a robotics curriculum at an academic general surgery training program: our initial experience. J Robot Surg. 2016;10(3):209-213.

31. Dias RD, Gupta A, Yule SJ. Using Machine Learning to Assess Physician Competence: A Systematic Review. Academic Medicine. 2019;94(3):427-439. 